\title{
Impact of Oil Polarity on the Mixing Time at the Pore Scale in Low Salinity Waterflooding
}

\author{
Saheb Mohammadi ${ }^{a}$, Hassan Mahani ${ }^{a,}{ }^{*}$, Shahab Ayatollahi ${ }^{a}$, Vahid Niasar $^{\mathrm{b}}$ \\ a Department of Chemical and Petroleum Engineering, Sharif University of Technology, Tehran, Iran. \\ ${ }^{b}$ Department of Chemical Engineering and Analytical Science, School of Engineering, University of Manchester, Manchester \\ M13 9PL, UK. \\ *Corresponding author email: hmahani@sharif.edu, mahani h@yahoo.com
}

\section{Supporting Information: Interfacial Tension (IFT) and Contract Angle Measurement as a Function of Dye (Food Coloring Agent) Concentration}

A bench-top, in-house built setup was used to measure IFT. A standard pendant drop method was used to measure IFT between the oil and the HSW brine at ambient temperature and pressure. The dye concentration in the brine was varied from $0 \%$ to 4 vol \%. Above 4 vol \% concentration, the brine solution was opaque which inhibited identification of oil-brine interface. Thus, IFT measurement was not possible. Dynamic IFT was measured as a function of time until reaching equilibrium. 3-5 measurements at each dye concentration were performed for reproducibility. The IFT error is $\pm 1 \mathrm{mN} / \mathrm{m}$. The equilibrium values have been reported in Table SI-1. The data shows a slight reduction in the IFT as dye concentration increases.

Table SI-1: Interfacial tension between crude oil and HSW (100,000 ppm NaCl) as a function of dye concentration

\begin{tabular}{|c|c|}
\hline $\begin{array}{c}\text { Dye concentration } \\
(\text { vol } \%)\end{array}$ & IFT $(\mathbf{m N} / \mathbf{m})$ \\
\hline 0 & 19.2 \\
\hline 1 & 18.1 \\
\hline 4 & 14.9 \\
\hline
\end{tabular}

Captive-drop method was used to measure the contact angle between the oil, HSW and the glass as a function of dye concentration. To conduct the test, a glass plate, made from the same material used for fabricating the micromodels, was cleaned consecutively with toluene, hydrochloric acid ( $\mathrm{HCl}$ ) and demineralized water to remove any contamination from the surface. The glass plate was placed in the intended brine for a few hours to equilibrate. Then an oil droplet was released from a U-shape capillary tube positioned about 2-3 mm underneath the glass surface. The oil droplet was then allowed to rest on the surface, age and reach steady-state. Within 1-2 days the contact angle reached the final equilibrium value. During the whole measurement high-resolution images were taken from the droplet. The images were processed with Digimizer v5.3.5 to determine the three-phase contact line and measure the contact angle as a function of time. The same procedure was followed at all dye concentrations. Each test was repeated three times and the average values have been reported in Table SI-2. Measurements at dye concentration above 6 vol \% was practically impossible. The data shows a minor change of wettability as dye concentration is increased to 4 vol \%. 
Table SI-2: Contact angle between crude oil, HSW (100,000 ppm NaCl) and glass as a function of dye concentration

\begin{tabular}{|c|c|}
\hline $\begin{array}{c}\text { Dye concentration } \\
(\text { vol \%) }\end{array}$ & $\begin{array}{c}\text { Contact angle (degrees) } \\
\text { - measured through the water phase }\end{array}$ \\
\hline 0 & 57.4 \\
\hline 1 & 62.8 \\
\hline 4 & 62.5 \\
\hline 6 & 67 \\
\hline
\end{tabular}

